\title{
Comparison of qualitative information obtained with the echocardiographic assessment using subcostal-only view and focused transthoracic echocardiography examinations: a prospective observational study
}

\section{Comparaison des informations qualitatives obtenues par évaluation échocardiographique à l'aide d'une unique vue sous- costale et d'examens ciblés d'échocardiographie transthoracique : une étude observationnelle prospective}

\author{
Nibras Bughrara, MD, FCCM, FASA $(1) \cdot$ J. Ross Renew, MD, FASA, FASE • \\ Kenneth Alabre, MD • Josh Schulman-Marcus, MD • \\ Krishnaveni Sirigaddi, MD • Aliaksei Pustavoitau, MD, MHS, FCCM • \\ Elizabeth R. Lesser, MS · Jose L. Diaz-Gomez, MD, FCCM, FASE
}

Received: 3 March 2021 /Revised: 5 October 2021 / Accepted: 6 October 2021 / Published online: 18 November 2021

(C) Canadian Anesthesiologists' Society 2021

\begin{abstract}
Purpose To evaluate whether echocardiographic assessment using the subcostal-only window (EASy) compared with focused transthoracic echocardiography (FTTE) using three windows (parasternal, apical, and subcostal) can provide critical information to serve as an entry-point technique for novice sonographers.
\end{abstract}

This article is accompanied by an editorial. Please see Can J Anesth 2022 ; this issue.

Supplementary Information The online version contains supplementary material available at https://doi.org/10.1007/s12630021-02152-6.

\footnotetext{
N. Bughrara, MD, FCCM, FASA ( $₫)$

Department of Anesthesiology, Albany Medical Center, MC-

131, 47 New Scotland Avenue, Albany, NY 12208, USA

e-mail: bughran@amc.edu

J. R. Renew, MD, FASA, FASE

Department of Anesthesiology and Perioperative Medicine,

Mayo Clinic College of Medicine, Jacksonville, FL, USA

K. Alabre, MD

Department of Anesthesiology, Brown/Rhode Island Hospital, Providence, RI, USA

J. Schulman-Marcus, MD

Department of Cardiology, Albany Medical College, Albany, NY, USA
}

Methods We conducted a retrospective study to compare diagnostic information acquired during EASy and FTTE examinations on qualitative left ventricular $(L V)$ size, $L V$ contractility, right ventricular $(R V)$ size, $R V$ contractility, interventricular septal position, and the presence of a significant pericardial effusion. Anesthesiology residents (novice users) performed FTTE for hemodynamic instability and/or respiratory distress or to define volume status in the perioperative setting, and later collected images were grouped into EASy and FTTE examinations. Both examinations were reviewed independently by a board-certified cardiologist and an anesthesiologist

KrishnaveniSirigaddi, MD

Department of Internal Medicine, Berkshire Medical Center, Pittsfield, MA, USA

A. Pustavoitau, MD, MHS, FCCM

Department of Anesthesiology, Johns Hopkins Hospital, Baltimore, MD, USA

\section{E. R. Lesser, MS}

Department of Health Sciences Research, Mayo Clinic College of Medicine, Jacksonville, FL, USA

J. L. Diaz-Gomez, MD, FCCM, FASE

Division of Cardiovascular Anesthesia and Critical Care Medicine, Baylor College of Medicine, Texas Heart Institute, Houston, TX, USA 
proficient in critical care echocardiography. FTTE and EASy findings were compared utilizing Gwet's ACl coefficient to consider disagreement due to chance.

Results We reviewed 102 patients who received FTTE over a period of 14 months. Of those, 82 had usable subcostal views and were included in the analysis. There was substantial agreement for qualitatively evaluating $R V$ size (Gwet's AC1, 0.70; 95\% confidence interval [CI], 0.54 to 0.85 ), LV size (Gwet's AC1, 0.73; 95\% CI, 0.58 to 0.88 ), and LV contractility (Gwet's AC1, 0.73; 95\% CI, 0.58 to 0.88) utilizing EASy and FTTE. Additionally, there was an almost perfect agreement when assessing the presence of pericardial effusion (Gwet's AC1, 0.98; 95\% CI, 0.95 to 1.0) and RV contractility (Gwet's AC1, 0.84; 95\% CI, 0.74 to 0.95) and evaluating the motion of the interventricular septum (Gwet's AC1, 0.92; 95\% CI, 0.85 to 0.99).

Conclusions When images could be obtained from the subcostal window (the EASy examination), qualitative diagnostic information was sufficiently accurate compared with information obtained during FTTE examination. Our findings suggest that the EASy examination can serve as the entry point technique to FTTE for novice clinicians.

\section{Résumé}

Objectif Déterminer si l'évaluation échocardiographique se fondant sur la fenêtre unique sous-costale (EASy) par rapport à une échocardiographie transthoracique ciblée (ETTC) fondée sur trois fenêtres (parasternale, apicale et sous-costale) pouvait fournir des informations critiques et servir de technique de départ pour enseigner l'échographie aux novices.

Méthode Nous avons réalisé une étude rétrospective afin de comparer les informations diagnostiques acquises lors des examens échocardiographiques EASy et ETTC concernant la taille qualitative $d u$ ventricule gauche $(V G)$, la contractilité $d u V G$, la taille du ventricule droit (VD), la contractilité du VD, la position septale interventriculaire et la présence d'un épanchement péricardique significatif. Les résidents en anesthésiologie (utilisateurs novices) ont réalisé une ETTC pour détecter une instabilité hémodynamique et / ou une détresse respiratoire ou pour définir l'état volémique dans un contexte périopératoire; par la suite les images colligées ont été regroupées en examens EASy et ETTC. Les deux examens ont été indépendamment passés en revue par un cardiologue certifié et un anesthésiologiste formé en échocardiographie de soins intensifs. Les résultats des examens d'ETTC et d'EASy ont été comparés en utilisant le coefficient ACl de Gwet pour tenir compte des désaccords dus au hasard.

Résultats Nous avons passé en revue 102 patients ayant reçu une ETTC sur une période de 14 mois. De ce nombre,
82 ont présenté des vues sous-costales utilisables qui ont été incluses dans l'analyse. Il y avait une importante concordance entre les examens EASy et ETTC pour évaluer qualitativement la taille $d u$ VD (ACl de Gwet, 0,70; intervalle de confiance [IC] à $95 \%, 0,54$ à 0,85$)$, la taille $d u$ VG (ACl de Gwet, 0,73; IC $95 \%, 0,58$ à 0,88) et la contractilité du VG (AC1 de Gwet, 0,73; IC $95 \%, 0,58$ à 0,88). De plus, il y avait une concordance quasi parfaite lors de l'évaluation de la présence d'épanchement péricardique (AC1 de Gwet, 0,98; IC $95 \%, 0,95$ à 1,0) et de la contractilité du VD (ACl de Gwet, 0,84; IC $95 \%$, 0,74 à 0,95 ) et de l'évaluation du mouvement du septum interventriculaire (ACl de Gwet, 0,92; IC $95 \%, 0,85$ à $0,99)$.

Conclusion Lorsque les images pouvaient être obtenues à partir de la fenêtre sous-costale (examen EASy), les informations diagnostiques qualitatives étaient suffisamment précises par rapport aux informations obtenues lors de l'examen d'ETTC. Nos résultats suggèrent que l'examen EASy peut servir de technique d'apprentissage précédant l'ETTC pour les cliniciens novices.

Keywords Echocardiography · Point-of-care ultrasound · Hemodynamic instability - Perioperative assessment . Anesthesiology training

Point-of-care ultrasound (POCUS) is commonly used in acute care settings. ${ }^{1-5}$ Emergency medicine physicians, intensivists, hospitalists, and anesthesiologists are using POCUS to address rapid changes in a patient's hemodynamic and/or respiratory status caused by pathologic process such as cardiac tamponade, hypovolemia, and acute ventricular systolic dysfunction. ${ }^{6-10}$

Focused transthoracic echocardiography (FTTE) is an application of POCUS, which evolved from comprehensive transthoracic echocardiography for urgent assessment of cardiopulmonary status. ${ }^{3}$ The FTTE examination typically comprises three windows that are identified based on the position of the ultrasound probe: parasternal, apical, and subcostal (Fig. 1). ${ }^{11}$ While primarily focusing on the heart, imaging the inferior vena cava (IVC) can also be performed from the subcostal window and provides important information during hemodynamic assessment. Cumulatively, the images obtained during FTTE can provide a rapid, qualitative, or semi-quantitative assessment of ventricular systolic function, myocardial wall motion abnormalities, gross valvular disease, pericardial effusion, volume status, and real-time fluid responsiveness. ${ }^{11}$ This technique is also useful as a timely 


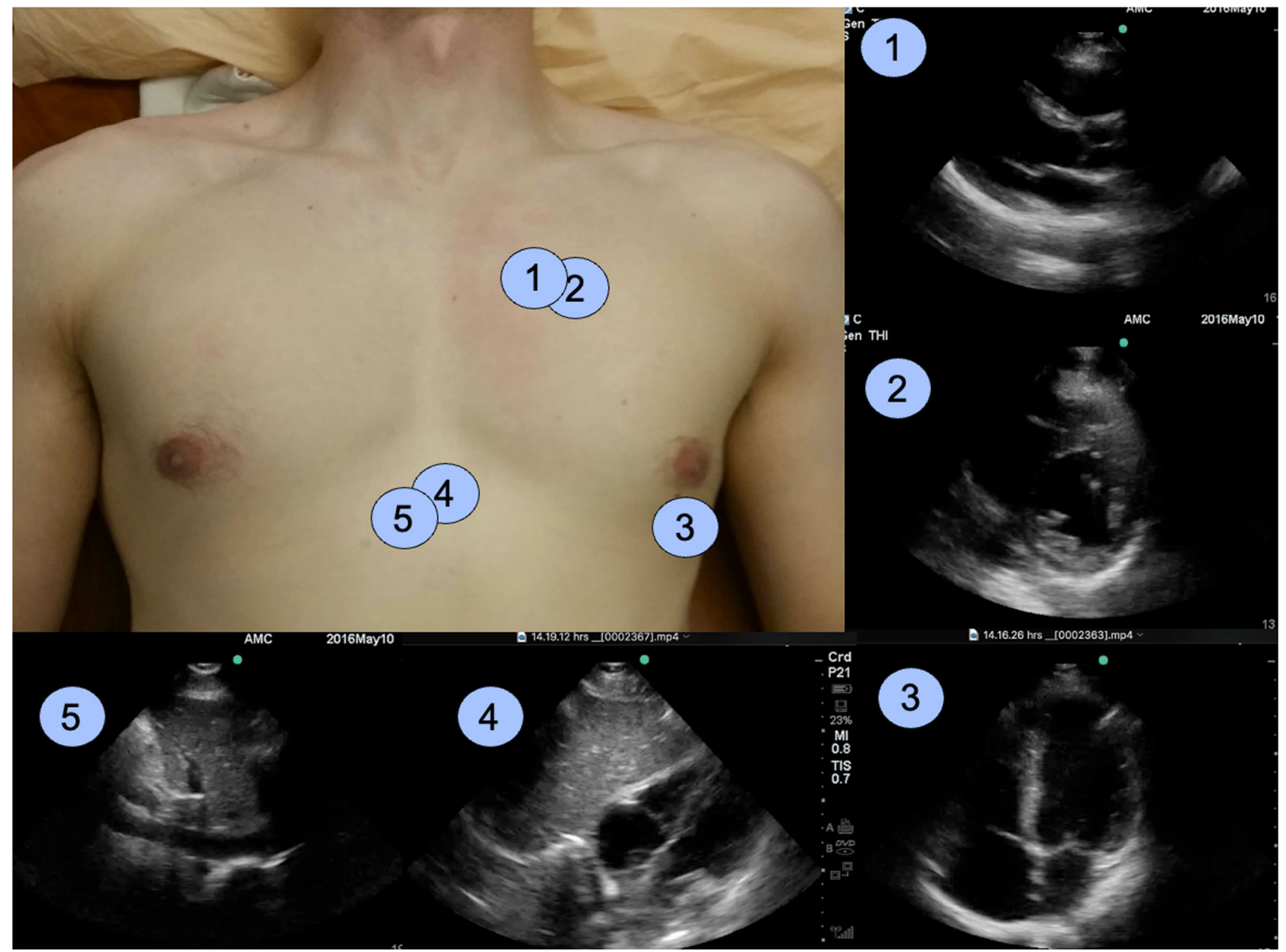

Fig. 1 FTTE includes five views obtained from three windows: (1) parasternal long axis, (2) parasternal short axis, (3) apical four chamber, (4) subcostal long access, (5) aubcostal IVC.

preoperative tool that can determine relevant information regarding the qualitative cardiac performance and has been shown to impact patient morbidity and mortality. ${ }^{8,12}$

The most common limiting factor for widespread use of FTTE remains the lack of expertise among clinicians; nevertheless, limited resources can also serve as a substantial barrier. ${ }^{13}$ While efforts have been made to investigate the most engaging and effective way to introduce FTTE to providers, ${ }^{14}$ adequate competence in this field became a core requirement in several specialties and remains an area of great interest. ${ }^{15}$

When patients are hemodynamically unstable and time constraints prohibit a comprehensive examination, obtaining just one view of the heart and IVC may prove to be a viable, time-saving approach (Electronic Supplementary Material [ESM], eVideo). ${ }^{16-18}$ In case series of patients with penetrating anterior chest trauma, the cardiac component of FAST examination (which includes the single subcostal cardiac view examination) was valuable in determination of traumatic pericardial effusion and effectively guided emergent surgical decisionmaking. ${ }^{19}$ In recent published case series, we demonstrated the feasibility of resident-performed EASy in advanced life support (EASy-ALS) and in the periresuscitative period. ${ }^{18}$ Consistent with previously reported protocols, ${ }^{16,17}$ we observed that teaching EASy-ALS simplifies the training by focusing on just one window during evaluation of patients with hemodynamic instability. ${ }^{18}$

While we previously demonstrated that findings of ventricular function, volume status, and pericardial effusion were consistent in $96 \%$ of patients with interpretable EASy images when immediately compared with FTTE by the supervised novice residents performing both examinations, such study design is prone to biased estimates. $^{20} \mathrm{We}$ designed the current study to compare diagnostic information acquired during EASy and FTTE examinations in the perioperative setting when images were independently interpreted by experts. 


\section{Methods}

\section{Patient population}

The Institutional Review Board (IRB) of Albany Medical Center approved this retrospective study. The requirement for written informed consent was waived by the IRB. We reviewed charts of all patients who received FTTE by anesthesiology residents. We included studies performed on perioperative patients over the age of $18 \mathrm{yr}$, located in the operating room, post-anesthesia care unit (PACU), and surgical intensive care unit, for whom both FTTE images and interpretation were available. Patients undergoing cardiac surgery were excluded. Indications for FTTE included: 1) hemodynamic instability defined by either hypotension with mean arterial blood pressure $<65 \mathrm{~mm}$ $\mathrm{Hg}$ or shock requiring vasoactive medications; 2) volume assessment for patients with oliguria defined by urine output $<0.5 \mathrm{~mL} \cdot \mathrm{kg}^{-1} \mathrm{hr}^{-1}$; 3) respiratory failure defined by acute decrease in oxygen saturation to $<90 \%$; 4) preoperative FTTE evaluation of American Society of Anesthesiologists functional class III and IV patients scheduled for major vascular and abdominal procedures upon request by attending anesthesiologist.

\section{Training requirements}

All residents completed a four-day course in FTTE and EASy during their postgraduate year 3 . The course was taken during PACU rotation and consisted of didactic lectures, hands-on training, and simulation. Didactic prerecorded lectures were provided through licensed webbased course by the Society of Critical Care Medicine. These lectures were moderated by faculty member, went over ultrasound physics and clinical applications, and were delivered during four-hour daily sessions over four days. Hands-on training included completion of 20 FTTE studies. Finally, all residents participated in four-hourlong high-fidelity team-based simulation training encompassing five pulseless electrical activity arrest scenarios: hypovolemia, massive myocardial infraction, massive pulmonary embolism, tamponade, and tension pneumothorax. Upon completion of training and achieving competency, residents were signed-off to perform EASy and FTTE without direct supervision but with mandatory review of each case. ${ }^{18}$

\section{Data collection}

Anesthesiology residents obtained the subcostal views first (EASy examination of the heart and IVC), followed by the apical and parasternal views to complete the FTTE. All images were obtained using an ultrasound machine (X-
Porte, FUJIFILM Sonosite, Inc., Bothell, WA, USA) equipped with a phased array transducer. Studies were interpreted by the resident directly supervised by a faculty member who was considered proficient in critical care ultrasound (i.e., had taken and passed the National Board of Echocardiography special competencies in critical care echocardiography examination in January 2019 and who used POCUS on a regular basis in the intensive care unit). Findings relevant to clinical care were shared in real-time with the patient's primary medical team. The entire examination was saved as FTTE examination and deidentified for the purpose of later review. Next, subcostal images of the heart and IVC were copied and saved in a separate file to represent EASy examination. At a later date, EASy and FTTE examinations were independently reviewed by a cardiologist (J.S.M.) certified in advanced echocardiography by N.B.E. and a critical care boardcertified anesthesiologist considered proficient in critical care ultrasound (N.B.). J.S.M. reviewed images from the EASy examination for the first 50 patients and images from the FTTE examination for the last 52 patients, while N.B. reviewed images from the FTTE examination for the first 50 patients and images from the EASy examination for the last 52 patients.

To evaluate quality of studies, we used a scoring system by assigning 0-2 points for each one of the six components of examination (pericardium, right ventricular [RV] size, $\mathrm{RV}$ function, interventricular septum, left ventricular [LV] size, and LV function) based on confidence of evaluator in observed findings. Zero points were assigned if evaluator was unable to assess the component of the examination; one point was assigned if there was incomplete view and additional views would be required for evaluation, and two points were assigned if there was complete view of the structure. Based on the total score the examination was graded as good (score 10-12), adequate (score 7-9), or poor (score 6 and below). Studies where subcostal views were of poor quality or documented as "unobtainable" were excluded from the analyses; nevertheless, the rest of the examination was completed and saved for further review.

During their review, J.S.M. and N.B. documented whether 1) a significant pericardial effusion was present (defined by a linear two-dimensional measurement of $>10$ $\mathrm{mm}$ present throughout the cardiac cycle); 2) the right and left ventricles were of normal dimension or enlarged using visual estimation; 3) the RV and LV systolic functions were subjectively normal or depressed; 4) the interventricular septum bowed to the left any time throughout the cardiac cycle as a sign of RV strain. Reviewers used the same interpretation form and taxonomy (ESM eAppendix). For the purpose of this study, all findings for each indication were classified on a binary 
scale (normal vs abnormal); the normal category included small and hyperdynamic ventricles. Discrepancies then were further examined to determine whether pathology missed was actionable enough to intervene.

\section{Statistical analysis}

After the review process, findings between EASy and FTTE were analyzed with Gwet's $\mathrm{AC}_{1}$ coefficient to consider disagreement due to chance. Corresponding 95\% confidence intervals were estimated by using the "gac" function from the "rel" package (with $\mathrm{R}$ software). Agreement measures range from 0 to 1 where coefficients $\leq 0.2$ are indicative of slight agreement, $0.21-0.40$ fair agreement, $0.41-0.60$ moderate agreement, $0.61-0.80$ substantial agreement, and $\geq 0.80$ almost perfect agreement according to benchmark scale originally provided for Cohen's kappa interpretation. ${ }^{21}$ Categorical variables were summarized with frequencies and percentages. Continuous variables were reported as mean (standard deviation) or median (interquartile range), as appropriate. All tests were two-sided, and $P$ values $<0.05$ were considered statistically significant. All analysis was performed in $\mathrm{R}$ Statistical Software (version 3.6.1; $\mathrm{R}$ Foundation for Statistical Computing, Vienna, Austria).

\section{Results}

All reviewed images were acquired by eight anesthesia residents (5-19 examinations per each resident), and we identified 102 patients who received FTTE from August 2017 to September 2018. The average time spent to acquire EASy was 4 min, and FTTE was 16 min. Image quality is described in Fig. 2. Twenty studies were excluded from the two experts' comparison because they were reported with a "poor" or "unobtainable" subcostal image. The two most common indications for examination were preoperative assessment and arterial hypotension (Table 1).

When reviewing the acquired images of the remaining 82 patients, the two independent reviewers displayed substantial overall agreement on the assessment of RV size (Gwet's AC1, 0.70; 95\% confidence interval [CI], 0.54 to 0.85 ), left ventricular (LV) size (Gwet's AC1, 0.73; 95\% CI, 0.58 to 0.88 ), and LV contractility (Gwet's AC1, 0.73; 95\% CI, 0.58 to 0.88 ) utilizing EASY and FTTE.

There was an almost perfect agreement when assessing the presence of pericardial effusion (Gwet's AC1, 0.98; 95\% CI, 0.95 to 1.0 ) and RV contractility (Gwet's AC1, $0.84 ; 95 \% \mathrm{CI}, 0.74$ to 0.95 ) and evaluating the motion of the interventricular septum (Gwet's AC1, 0.92; 95\% CI, 0.85 to 0.99 ). There was moderate agreement between the two reviewers when rating the image quality for the EASy and FTTE examinations as 'good', 'adequate', and 'poor' (weighted Gwet's AC1, 0.52; 95\% CI, 0.38 to 0.67) (Table 2).

In two cases actionable findings were missed by EASy: 1) LV function assessed as normal in EASy was categorized as severely depressed by FTTE. This patient had morbid obesity (body mass index, $45 \mathrm{~kg} \cdot \mathrm{m}^{-2}$ ), and only basal segments of the LV were visualized well in the subcostal view. Based on FTTE LV function was classified as severely depressed consistent with Takotsubo cardiomyopathy with its key echocardiographic features of symmetrical circumferential regional dilation and dysfunction of the mid-ventricular and apical area, with preservation of the basal segments; 2) RV size assessed as normal on EASy, while being classified as severely dilated on FTTE. Upon further investigation such discrepancy was determined to be due to technique related to probe rotation.

\section{Discussion}

In the current study, we found substantial agreement between EASy and FTTE for qualitative assessment of ventricular size and LV systolic function as determined by two independent reviewers. Additionally, there was almost perfect agreement when assessing the presence of pericardial effusion, RV systolic function, and motion of the interventricular septum as surrogate for RV dysfunction. Regardless of the specific finding being evaluated, such level of agreement suggests that focusing on and obtaining images of the heart and IVC from a single window (subcostal) can provide sufficient qualitative diagnostic information in patients with hemodynamic instability compared with FTTE images acquired over three windows.

While the EASy examination can obtain similar information as the FTTE examination, it has several advantages over its more comprehensive counterpart. First, focusing on one window allows the novice echocardiographer such as anesthesiology residents to gain the basic competence in acquiring subcostal views while recognizing the sonographic anatomy, qualitative findings, and screening for overt pathology. Second, performing the EASy examination is faster than performing the FTTE examination as the clinician only needs to place the probe over one echocardiographic window. ${ }^{20}$ Third, the subcostal window allows for imaging of the IVC, with small and collapsible IVC suggestive of hypovolemia and plethoric IVC suggestive of hypervolemia or obstructive physiology. When combined with upper lung views to look for fluid tolerance the entire examination can take only 3 to $4 \mathrm{~min}$ and provide important clinical information that could determine the 

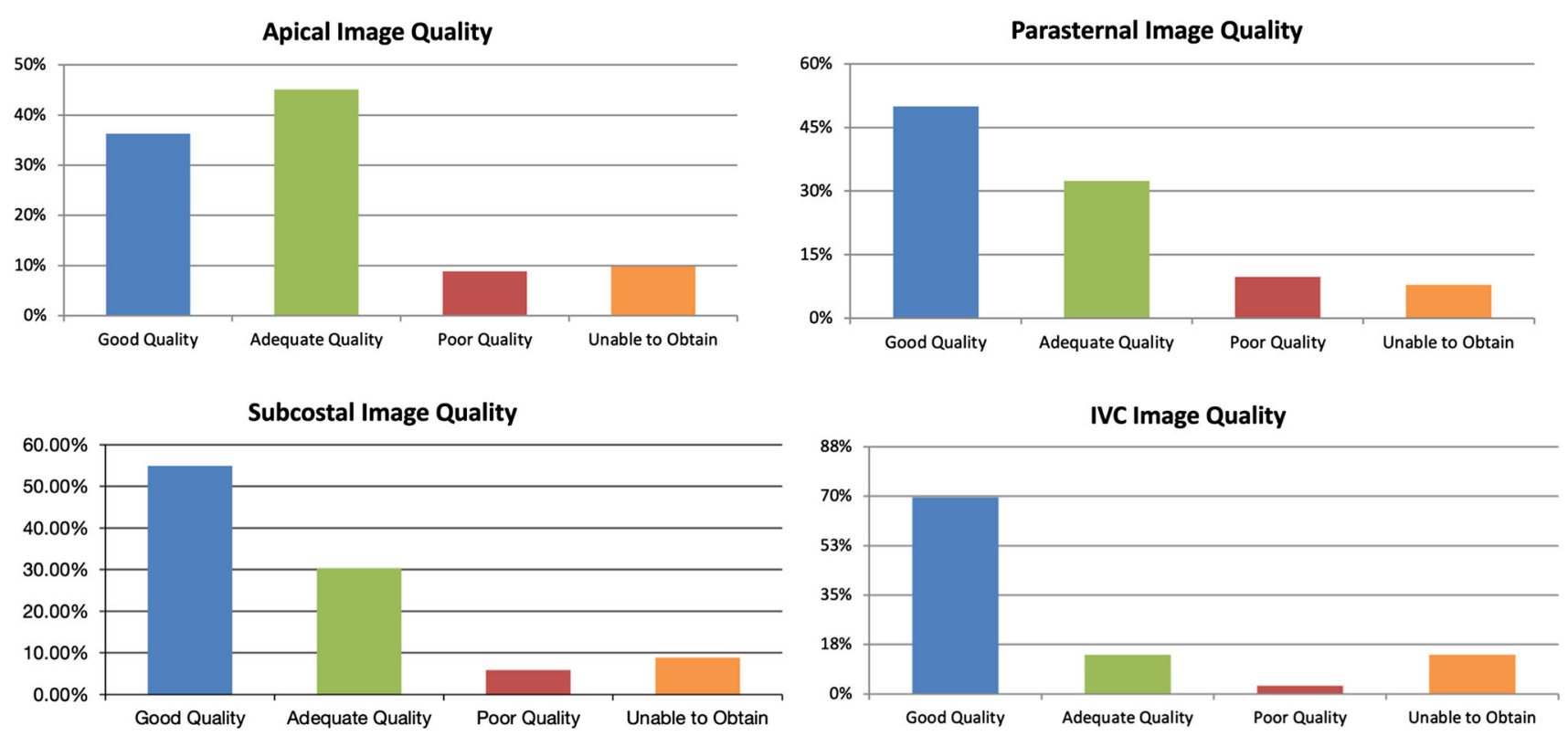

Fig. 2 FTTE examinations performed on 102 consecutive patients in postanesthesia care unit (PACU), surgical intensive care unit (SICU), and the operating rooms (OR); $96.9 \%$ revealed useful images that contributed positively to evaluation (at least one interpretable window).

Table 1 Baseline characteristics of patients and indications for examination

\begin{tabular}{lr}
\hline Age $(\mathrm{yr})$, mean $(\mathrm{SD})$ & $65(13) \_$ \\
\hline Female sex, $n(\%)$ & $48(47 \%)$ \\
Body mass index $\left(\mathrm{kg} \cdot \mathrm{m}^{-2}\right)$, mean $(\mathrm{SD})$ & $28(8)$ \\
Indication for exam & $31(30 \%)$ \\
$\quad$ Hypotension, $n(\%)$ & $18(18 \%)$ \\
Volume status, $n(\%)$ & $8(8 \%)$ \\
Respiratory distress, $n(\%)$ & $10(10 \%)$ \\
Cardiac arrest, $n(\%)$ & $35(34 \%)$ \\
Preoperative assessment $n(\%)$ &
\end{tabular}

Total $N=102$. Sex was determined by patient-reported designation $\mathrm{SD}=$ standard deviation

etiology of shock. ${ }^{22}$ A simple approach that focuses on one window can serve as the first step to achieve the basic competency in POCUS for both trainees and practicing clinicians. While mastering the EASy examination would not serve as the final destination of competence in POCUS, it can help clinicians gain familiarity and enhance visuospatial orientation and technical skills in urgent situations and overall confidence in employing this imaging modality during cardiac arrest. ${ }^{18}$

Our study has several limitations. Interpretable images were obtained in $80 \%$ of patients compared with FTTE (97\%); nevertheless, many patients could be screened by novices rapidly leaving only $20 \%$ that require a more experienced person to perform FTTE. The novelty of comparing two echocardiography examinations precluded the authors from performing a formal power analysis and determining the optimal number of examinations that should be completed to determine the level of agreement. The reporting system utilized during the current study is simplistic and does not allow for describing nuanced variation in such findings. Nevertheless, this basic reporting structure was utilized in an attempt to replicate the qualitative reports that novice echocardiographers may use in real-world settings. Additionally, while we are studying the agreement between two echocardiographic techniques, our results are certainly dependent upon whether FTTE had an impact on management of patient care. To minimize this phenomenon, the majority $(66 \%)$ of the reviewed examinations involved clinical scenarios where echocardiography has traditionally been described 
Table 2 Summary of agreement between cardiologist and anesthesiologist

\begin{tabular}{|c|c|c|c|}
\hline & $\begin{array}{l}\text { EASy by cardiologist } \& \\
\text { FTTE by anesthesiologist }\end{array}$ & $\begin{array}{l}\text { FTTE by cardiologist } \& \\
\text { EASy by anesthesiologist }\end{array}$ & Overall \\
\hline \multicolumn{4}{|l|}{ Pericardial effusion } \\
\hline$N$ & 45 & 37 & 82 \\
\hline Gwet's AC1 (95\% CI): & $0.97(0.91$ to 1.0$)$ & $1.0(1.0$ to 1.0$)$ & $0.98(0.95$ to 1.0$)$ \\
\hline Agree & $44(98 \%)$ & $37(100 \%)$ & $81(99 \%)$ \\
\hline Present & $7(16 \%)$ & $7(19 \%)$ & $14(17 \%)$ \\
\hline Not present & $37(84 \%)$ & $30(81 \%)$ & $67(83 \%)$ \\
\hline \multicolumn{4}{|l|}{ RV size } \\
\hline$N$ & 45 & 37 & 82 \\
\hline Gwet's AC1 (95\% CI): & 0.81 (0.65 to 0.97$)$ & $0.54(0.24$ to 0.83$)$ & $0.70(0.54$ to 0.85$)$ \\
\hline Agree & $39(87 \%)$ & $27(73.0 \%)$ & $66(80 \%)$ \\
\hline Normal & $34(87 \%)$ & $21(78 \%)$ & $55(83 \%)$ \\
\hline Enlarged & $5(13 \%)$ & $6(22 \%)$ & $11(17 \%)$ \\
\hline \multicolumn{4}{|l|}{ RV contractility } \\
\hline$N$ & 45 & 37 & 82 \\
\hline Gwet's AC1 (95\% CI): & 0.83 (0.68 to 0.98$)$ & $0.86(0.71$ to 1.00$)$ & $0.84(0.74$ to 0.95$)$ \\
\hline Agree & $39(87 \%)$ & $32(86 \%)$ & $71(87 \%)$ \\
\hline Good & $36(92 \%)$ & $29(91 \%)$ & $65(91 \%)$ \\
\hline Depressed & $3(8 \%)$ & $3(9 \%)$ & $6(8 \%)$ \\
\hline \multicolumn{4}{|l|}{ Interventricular septum } \\
\hline$N$ & 45 & 37 & 82 \\
\hline Gwet's AC1 (95\% CI): & $0.93(0.84$ to 1.0$)$ & $0.91(0.79$ to 1.0$)$ & $0.92(0.85$ to 0.99$)$ \\
\hline Agree & $42(93 \%)$ & $34(92 \%)$ & $76(93 \%)$ \\
\hline No bounce & $42(100 \%)$ & $33(97 \%)$ & $75(99 \%)$ \\
\hline Bounce & $0(0 \%)$ & $1(3 \%)$ & $1(1 \%)$ \\
\hline \multicolumn{4}{|l|}{ LV size } \\
\hline$N$ & 45 & 37 & 82 \\
\hline Gwet's AC1 (95\% CI): & $0.74(0.54$ to 0.94$)$ & $0.72(0.50$ to 0.94$)$ & $0.73(0.58$ to 0.88$)$ \\
\hline Agree & $38(84 \%)$ & $30(81 \%)$ & $68(83 \%)$ \\
\hline Normal & $29(76 \%)$ & $26(87 \%)$ & $55(81 \%)$ \\
\hline Enlarged & $9(24 \%)$ & $4(13 \%)$ & $13(19 \%)$ \\
\hline \multicolumn{4}{|l|}{ LV contractility } \\
\hline$N$ & 45 & 37 & 82 \\
\hline Gwet's AC1 (95\% CI): & $0.61(0.37$ to 0.85$)$ & $0.86(0.70$ to 1.0$)$ & $0.73(0.58$ to 0.88$)$ \\
\hline Agree & $35(78 \%)$ & $34(92 \%)$ & $69(84 \%)$ \\
\hline Good & $26(74 \%)$ & $25(73 \%)$ & $51(74 \%)$ \\
\hline Depressed & $9(26 \%)$ & $9(26 \%)$ & $18(22 \%)$ \\
\hline \multicolumn{4}{|l|}{ Image quality } \\
\hline$N$ & 45 & 37 & 82 \\
\hline Weighted Gwet's AC1 (95\% CI): & 0.26 (N/A to 0.55$)$ & $0.56(0.34$ to 0.78$)$ & $0.52(0.38$ to 0.67$)$ \\
\hline Agree & $28(62 \%)$ & $25(68 \%)$ & $53(65 \%)$ \\
\hline Good & $17(61 \%)$ & $12(48 \%)$ & $29(55 \%)$ \\
\hline Adequate & $11(39 \%)$ & $13(52 \%)$ & $24(45 \%)$ \\
\hline Poor & $0(0 \%)$ & $0(0 \%)$ & $0(0 \%)$ \\
\hline Unable to decipher & $0(0 \%)$ & $0(0 \%)$ & $0(0 \%)$ \\
\hline
\end{tabular}

CI = confidence interval; EASy = echocardiographic assessment using subcostal only view; FTTE = focused transthoracic echocardiography; $\mathrm{N} / \mathrm{A}=$ not calculable; $\mathrm{RV}=$ right ventricle; $\mathrm{LV}=$ left ventricle 
as having direct clinical impact (i.e., hypotension, assessing volume status, and cardiopulmonary distress). As described, EASy can also miss certain pathologies due to limitations of the technique. To overcome these limitations, subcostal short axis view of the heart can be obtained when LV is not well visualized and special attention paid to visualize RV by tilting ultrasound probe to scan through the entire RV.

One limitation of the study is that image interpretation by novice sonographers was not examined. Since initial interpretation at the bedside was done together with the supervising anesthesiologist, this would provide biased estimates. Nevertheless, we were able to assess whether novice sonographers collected images sufficient to support clinical decision-making, as the ability of novice sonographers to collect high-quality images has implications for telemedicine, under conditions of massive influx of critically ill patients (e.g., patients with coronavirus disease), where residents can obtain EASy examinations as part of patient evaluation and then an expert interprets images during rounds or from home when on call. Further studies should compare novices' interpretation of EASy to experts' interpretation of FTTE.

In summary, when EASy examination can be obtained, it provides diagnostic information sufficient to aid in the assessment and management of patients with cardiopulmonary derangements in perioperative and critical care settings. Emphasizing and mastering one window can make echocardiography more accessible and appealing for any medical trainees or clinicians who are unfamiliar with this imaging modality. Further investigations are needed to measure the clinical impact that the EASy examination can have on patient outcomes.

Author contributions Nibras Bughrara participated in writing the protocol of the study, data collection, data analysis, and writing the manuscript. J. Ross Renew participated in writing the protocol, data analysis, and writing the manuscript. Kenneth Alabre participated in data collection and analysis and writing the manuscript. Josh Schulman-Marcus and Krishnaveni Sirigaddi participated in data collection and analysis. Aliaksei Pustavoitau participated in writing and editing the manuscript. Elizabeth R. Lesser participated in data analysis and writing the manuscript. Jose L. Diaz-Gomez participated in writing the protocol and editing the manuscript.

\section{Disclosure None.}

Funding statement None, support was provided solely by institutional and/or departmental sources.

Editorial responsibility This submission was handled by Dr. Philip M. Jones, Deputy Editor-in-Chief, Canadian Journal of Anesthesial Journal canadien d'anesthésie.

\section{References}

1. Neskovic AN, Edvardsen T, Galderisi M, et al. Focus cardiac ultrasound: the European Association of Cardiovascular Imaging viewpoint. Eur Heart J Cardiovasc Imaging 2014; 15: 956-60.

2. Via G, Hussain A, Wells $M$, et al. International evidence-based recommendations for focused cardiac ultrasound. J Am Soc Echocardiogr 2014; 27: 683.e1-33.

3. Labovitz AJ, Noble VE, Bierig $M$, et al. Focused cardiac ultrasound in the emergent setting: a consensus statement of the American Society of Echocardiography and American College of Emergency Physicians. J Am Soc Echocardiogr 2010; 23: 1225-30.

4. Moore CL, Copel JA. Point-of-care ultrasonography. N Engl J Med 2011; 364: 749-57.

5. Neskovic AN, Hagendorff A, Lancellotti P, et al. Emergency echocardiography: the European Association of Cardiovascular Imaging recommendations. Eur Heart $\mathrm{J}$ Cardiovasc Imaging 2013; 141: 1-11.

6. Oren-Grinberg A, Talmor D, Brown SM. Focused critical care echocardiography. Crit Care Med 2013; 41: 2618-26.

7. Spencer KT, Kimura BJ, Korcarz CE, Pellikka PA, Rahko PS, Siegel RJ. Focused cardiac ultrasound: recommendations from the American Society of Echocardiography. J Am Soc Echocardiogr 2013; 26: 567-81.

8. Canty DJ, Heiberg J, Yang Y, et al. Pilot multi-centre randomised trial of the impact of pre-operative focused cardiac ultrasound on mortality and morbidity in patients having surgery for femoral neck fractures (ECHONOF-2 pilot). Anaesthesia 2018; 73: 428-37.

9. Zhang J, Critchley LA. Inferior vena cava ultrasonography before general anesthesia can predict hypotension after induction. Anesthesiology 2016; 124: 580-9.

10. Diaz-Gomez JL, Renew JR, Ratzlaff RA, Ramakrishna H, Torp K, Via $G$. Can lung ultrasound be the first-line tool for evaluation of intraoperative hypoxemia? Anesth Analg 2018; 126: 2146-7.

11. Jensen $M B$, Sloth E, Larsen KM, Schmidt MB. Transthoracic echocardiography for cardiopulmonary monitoring in intensive care. Eur J Anaesthesiol 2004; 21: 700-7.

12. Canty DJ, Royse CF, Kilpatrick D, Bowyer A, Royse AG. The impact on cardiac diagnosis and mortality of focused transthoracic echocardiography in hip fracture surgery patients with increased risk of cardiac disease: a retrospective cohort study. Anaesthesia 2012; 67: 1202-9.

13. Sternschein RM, Gay EB, Palmer LJ, Hayes MM. Scanning for experts: practical approaches to incorporate ultrasound use in the intensive care unit and enhance an ultrasound educational program. Ann Am Thorac Soc 2019; 16: 1488-91.

14. Diaz-Gomez JL, Perez-Protto S, Hargrave J, et al. Impact of a focused transthoracic echocardiography training course for rescue applications among anesthesiology and critical care medicine practitioners: a prospective study. J Cardiothorac Vasc Anesth 2015; 29: 576-81.

15. Wiegers SE, Ryan T, Arrighi JA, et al. 2019 ACC/AHA/ASE Advanced Training Statement on Echocardiography (Revision of the 2003 ACC/AHA Clinical Competence Statement on Echocardiography): a report of the ACC Competency Management Committee. J Am Coll Cardiol 2019; 74: 377-402.

16. Niendorff DF, Rassias AJ, Palac R, Beach ML, Costa S, Greenberg M. Rapid cardiac ultrasound of inpatients suffering PEA arrest performed by nonexpert sonographers. Resuscitation 2005; 67: 81-7.

17. Hernandez C, Shuler K, Hannan H, Sonyika C, Likourezos A, Marshall J. C.A.U.S.E.: cardiac arrest ultra-sound exam-a better 
approach to managing patients in primary non-arrhythmogenic cardiac arrest. Resuscitation 2008; 76: 198-206.

18. Bughrara N, Herrick SL, Leimer E, Sirigaddi K, Roberts $K$, Pustavoitau A. Focused cardiac ultrasound and the periresuscitative period: a case series of resident-performed echocardiographic assessment using subcostal-only view in advanced life support. A A Pract 2020; DOI: https://doi.org/10. 1213/XAA.0000000000001278.

19. Tayal VS, Beatty MA, Marx JA, Tomaszewski CA, Thomason MH. FAST (focused assessment with sonography in trauma) accurate for cardiac and intraperitoneal injury in penetrating anterior chest trauma. J Ultrasound Med 2004; 23: 467-72.

20. Bughrara NF, Emr KS, Renew JR, Diaz-Gomez JL, Safa RN, Roberts $K$. Echocardiographic assessment using subxiphoid-only view (EASY) compared to focused transthoracic echocardiography (FOTE): a multicenter prospective study E. Anesthesiology 2018; A3089 (abstract).

21. Landis JR, Koch GG. The measurement of observer agreement for categorical data. Biometrics 1977; 33: 159-74.

22. Bughrara N, Diaz-Gomez JL, Pustavoitau A. Perioperative management of patient with sepsis and septic shock, part II: ultrasound support for resuscitation. Anesthesiol Clin 2020; 38: 123-34.

Publisher's Note Springer Nature remains neutral with regard to jurisdictional claims in published maps and institutional affiliations. 